\title{
Individuals with hyperphagia can voluntarily fast: Experience from Prader-Willi Syndrome
}

\author{
Fortu Benarroch ${ }^{1,2^{*}}$, Yehuda Pollak ${ }^{2}$, Maayan Wertman ${ }^{2}$, Harry J. Hirsch ${ }^{2}$, Larry Genstil ${ }^{2}$, \\ Varda Gross-Tsur ${ }^{2}$ \\ ${ }^{1}$ Herman Dana Division of Child and Adolescent Psychiatry, Hadassah-Hebrew University Medical Center, Jerusalem, Israel; \\ *Corresponding Author: fortuben@hadassah.org.il \\ ${ }^{2}$ PWS Multidisciplinary Clinic, Neuropediatric Unit, Shaare Zedek Medical Center, Jerusalem, Israel
}

Received 11 November 2012; revised 14 December 2012; accepted 23 December 2012

\section{ABSTRACT}

Relentless pursuit of food is a major characteristic of Prader-Willi Syndrome (PWS). We observed voluntary fasting among PWS individuals during a religious fast. Understanding the mechanisms involved in successful fasting could be an important contribution in developing more effective treatment of this syndrome. We conducted a prospective study to assess whether genotype, motivational attitudes (e.g. religiosity) and control patterns (e.g. different eating habits) would correlate with ability to fast. Among all individuals with PWS in Israel, 32 met inclusion criteria. Prior to the fast, each participant and parents/caregivers were interviewed for demographic, medical and behavioral data and completed questionnaires assessing motivational and control factors. 22 participants completed the fast. This ability was not accounted for by religiosity, demographic, medical variables or genetic subtype. This prospective study documents that in spite of extreme hyperphagia, adolescents and adults with PWS can voluntarily abstain from food for 25 hours; our findings suggest that they are able to activate mechanisms which improve their control of eating for a longer period than expected. The observation that the degree of religiosity did not impact on the ability to fast suggests that these mechanisms may be applicable to a wider range of circumstances and populations. The ability for selfcontrol under special circumstances deserves further study; it may be relevant to other types of severe obesity and possibly lead to improved methods of behavioral modification.

Keywords: Prader-Willi Syndrome; Fast; Obesity; Eating Control; Motivation

\section{INTRODUCTION}

Prader-Willi syndrome (PWS) is a neurogenetic, multisystemic disorder caused by a deletion or impaired expression of paternal genes in the 15q11-q13 region of chromosome 15. The incidence ranges from 1:10,000 to 1:30,000 live births, with equal gender and ethnic distribution [1]. PWS is characterized by multiple physical and neurobehavioral problems such as dysmorphic features, learning disabilities and severe maladaptive behaviors. The most striking, characteristic symptom however, is hyperphagia, with devastating effects on body weight, health and quality of life. If unrestricted, persons with PWS can eat enormous quantities of food and when regular food is unavailable, they will eat frozen, raw, or even pet food. Food is obtained by night raids to the kitchen, stealing from others' lunch bags and salvaging food from garbage. Begging, lying, stealing and breaking locks are not unheard of. Together with their low metabolic rate, this behavior leads to morbid obesity in almost every patient who is not under continuous supervision [1,2]. To date, there is no effective medication to ameliorate this eating problem. Its management requires 24-hour-a-day supervision, restrictive measures, planned physical activity and a strict diet divided into consistently scheduled meals [3].

Since food restriction management is a major challenge in treating PWS individuals, we were very surprised to find that many of the adolescents and adults with genetically diagnosed PWS voluntarily fast on the Jewish holiday, Yom Kippur, "the Day of Atonement”. Yom Kippur is a day of self-reckoning, in which approximately two-thirds of healthy individuals in Israel above the age of 12 - 13 years fast for 25 hours [4]. According to Jewish religious law, individuals with health problems, including those with PWS, are exempt from fasting. This paradoxical behavior cannot be easily explained on the basis of religious motivation since many of the individuals with PWS who fast are secular and those who are re- 
ligious will, on other occasions, transgress other food-related religious laws. Moreover, many live in residential homes where meals are served on Yom Kippur with no encouragement to fast. Studying the factors contributing to successful fasting on Yom Kippur could lead to a better understanding of food-related behavior in PWS.

We undertook a prospective study of fasting in individuals with PWS, hypothesizing that there is a correlation between the ability to fast, genetic subtype and two main groups of factors: motivational factors (e.g. religiosity and social desirability) and control factors (e.g. self-control, eating habits).

\section{METHODS AND PROCEDURES}

\subsection{Participants}

The national multidisciplinary PWS clinic at Shaare Zedek Medical Center treats nearly all (100/106) individuals with a genetic diagnosis of PWS in Israel. We approached all those who fulfilled the inclusion criteria:

- Jewish males over 13 years of age and females over 12 years (minimal age for religious obligation to fast on Yom Kippur).

- Individuals able to understand the self report questionnaires.

- An expressed wish to fast on the upcoming Yom Kippur, either a verbal declaration before the fast or practical attempts to fast.

Permission for the study was received from Shaarei Zedeck Medical Center ethics committee (IRB).

Among the 100 individuals in the clinic, 41 met inclusion criteria. Four refused to participate in the study; two others, it turned out later, had had no intention to fast and three were excluded for medical reasons (e.g. diabetes). The participants or guardians signed the consent forms. Among the 32 participants, 10 live at home and 22 in residential facilities. The characteristics of individuals with PWS who intended to fast are shown in Table 1.

\subsection{Protocol and Tools}

During the three weeks preceding the fast, each individual with PWS and parent (if living at home) or caregiver (if living in residential facility) completed questionnaires, assisted, when necessary, by one of the researchers (M.W.).

\subsubsection{Data Collected from Caregivers before the Fast}

1) Physical and demographic data questionnaire including age, body weight and height, Body Mass Index (BMI), fluctuations in body weight during the last year, for hostel dwellers, elevations in body weight during vacations at home and length of living in hostel and six
Table 1. Characteristics of individuals with PWS who intended to fast on Yom Kippur.

\begin{tabular}{cccc}
\hline \multirow{2}{*}{ Age (years) } & Mean (SD) & Range & \\
& $21.6(5.9)$ & $12-32$ & \\
Gender & Females $\mathrm{n}=15$ & Males $\mathrm{n}=17$ & \\
& $(46.9 \%)$ & $(53.1 \%)$ & \\
Genotype & Del $\mathrm{n}=19$ & UPD $\mathrm{n}=12$ & ICD $\mathrm{n}=1$ \\
& $(59.4 \%)$ & $(37.5 \%)$ & $(3.1 \%)$ \\
BMI & Mean (SD) & Range & \\
& $29.8(7.9)$ & $19.1-47.8$ & \\
\hline
\end{tabular}

Del: deletion; UPD: uniparental disomy; ICD: imprinting center defect.

dichotomic categories: gender, genotype [uniparental dysomy (UPD) vs deletion (Del) according to genetic diagnosis], growth hormone (GH) therapy any time during life, accommodation (home vs hostel), religiosity and receiving psychotropic medication during the study.

2) "PWS behavioral characteristics questionnaire" (PWS-BCQ) [3]: This 38-item questionnaire was developed by our PWS team to quantify the main behavioral aspects of PWS and is organized in five clusters: abnormal emotional regulation, food-seeking related behaviors, mental inflexibility, oppositionality and interpersonal problems/self-injury. Each item is rated on a $0-2$ scale of frequency $(0=$ seldom, $1=$ sometimes true, $2=$ often $)$.

3) "Possible mediators of the capacity to fast" (PMCF) is a semi-structured interview, developed for this study, to assess eight factors hypothesized as potential mediators of the ability to control the drive to eat and to fast: intensity of the drive to eat, strength of the motivation to fast, social desirability, responsiveness to concrete reinforcements, stubbornness, passivity, religiosity, and in the case of a religious upbringing, the ability to obey other food related restrictions. Answers were given on a 5 points scale of intensity.

4) Three Factor Eating Questionnaire-Revised 18 (TFEQ-R18) [5]: This questionnaire consists of three factors: “Cognitive Restraint” (e.g. "I deliberately take small helpings as a means of controlling my weight"), "Uncontrolled Eating” (e.g. "When I see a real delicacy, I often get so hungry that I have to eat right away") and "Emotional Eating" (e.g. "When I feel anxious, I find myself eating").

5) The "Effortful Control" factor (ECF) of the short form of the Adult Temperament Questionnaire [6]. This factor consists of 3 subscales: “Activation Control” (e.g. "I can keep performing a task even when I would rather not do it"); "Inhibitory Control” (e.g. "I can easily resist talking out of turn, even when I'm excited and want to express an idea"); and "Attention" (e.g. "When interrupted or distracted, I usually can easily shift my attention back to whatever I was doing before"). Questions are rated on a 1 - 7 Likert scale.

6) Child Behavior Checklist (CBCL) Hebrew version 
[7]: This questionnaire consists of 118 questions, rated on a 0 - 2 scale $(0=$ not true, $1=$ sometimes true, 2 = true). The CBCL yields eight narrow-band scales: Withdrawn, Somatic Complaints, Anxious/Depressed, Social Problems, Thought Problems, Attention Problems, Delinquent Behavior, and Aggressive Behavior.

7) Behavior Rating Inventory of Executive Function (BRIEF) [8]. In the 86-item parent version used in this study, answers are given on a three-point scale. The 86 items result in three indices (Global Executive Composite, Metacognitive Index and Behavioral Regulation index), and eight subscales (Inhibition, Shifting, Emotional Control, Initiating, Working Memory, Planning/Organizing, Organizing of Material and Monitoring). The Hebrew version has adequate reliability $(\alpha=0.76-0.91)[9,10]$.

\subsubsection{Data Collected from the Individuals with PWS before the Fast}

1) The self-report version of the PMCF semi-structured interview described above.

2) The self-report version of the TFEQ-R18.

3) The "Shapiro Control Inventory" (SCI) [11]: This is a reliable and valid measure of perceived sense of control. The original inventory includes 187 items that are scored on ten control scales. For the purpose of the current study we used only three scales (37 questions): "Negative Sense of Control” (e.g. "I am too passive and helpless"), "Positive Sense of Control" (e.g. "I have a positive sense of control in my life") and "Desire for Control" (e.g. "I have a great desire to be in control”).

The original English versions of the TFEQ-R18, the ECF and the SCI were translated into Hebrew using multiple translators and independent back-translations.

\subsubsection{Data Collected within a Week Following the Fast}

The participants and caregivers were asked whether the full 25-hour fast was successfully completed. All PWS individuals in our study are under 24 hours a day supervision by their parents or caregivers as part of their regular management, and uncontrolled access to food in their environment is very difficult. Therefore, self-reporting of successful fasting was easily confirmed by the parents or caregivers, allowing for reliable ascertainment. Also, since PWS behavior is highly sensitive to contingency rewards, the interview took into account this possibility. Caregivers reported that they did not promise any reward for fasting, the meal at the end of the fast was a regular dinner and no presents were given to those who finished the fast.

\section{RESULTS}

From the 32 individuals in our sample, 22 completed the 25 hours fast and comprise the study group. Three participants fasted for an average of 18 hours but were excluded from the analyses since they did not complete the fast. Seven participants started to fast but resumed eating within a few hours ( 1 - 10 hours) and comprise the control group. Statistical analyses are based on comparisons between these 2 groups unless stated otherwise. The dependent measures listed above were calculated and compared between the two groups using t- and chisquare tests corrected for multiple comparisons. Demographic variables were covariated.

None of the demographic or medical parameters (Table 1) were significantly associated with fasting. This included gender ( $\mathrm{p}<0.59)$, age $(0.60)$, genotype $(0.92)$, home vs hostel (0.37), body weight (0.79), BMI (0.27), fluctuations in body weight during the previous year (0.74), for hostel dwellers-elevations in body weight during vacations at home (0.20) and length of living in hostel (0.79), religiosity (0.50), medication usage $(0.55)$ and GH therapy (0.07).

There were no significant associations between predictors of fasting on the PMCF interview and the ability to fast. The one exception was the rating given by the caregivers' perception of the importance of the fast for each individual which was greater $(p<0.014)$ for those who completed the fast.

MANOVA on all the questionnaires (PWS-BCQ, CBCL, TFEQ-R18, BRIEF and ECF) revealed no significant differences between fasting and non-fasting participants ( $p$ $<0.69,0.18,0.54,0.45,0.33$, respectively). Post-hoc analyses revealed that those who completed the fast had higher tolerance for pain ( $\mathrm{p}<0.03$ ) on the PWS-BCQ and had lower scores on the "anxious/depressed" subscale ( $p$ $<0.017)$ of the CBCL.

MANOVA on the self-reported TFEQ-R18 and SCI scales resulted in marginal effect $(\mathrm{p}<0.09,0.07$, respecttively). Univariate ANOVA on each of the scales showed that participants who completed the fast felt better self control, as seen by significantly lower scores on "uncontrolled eating (TFEQ-R18)" ( $\mathrm{p}<0.02)$ and lower "negative sense of control (SCI)" ( $p<0.02)$.

\section{DISCUSSION}

It was surprising to find that the majority (22/29) of adolescents and adults with PWS in this national cohort who expressed an interest in fasting on Yom Kippur were able to complete the 25-hour fast. Despite having the typical PWS eating phenotype, these individuals were able to refrain from eating for the duration of the one day fast and without receiving any particular reward.

We found no correlation with genetic subtype, a finding which does not support the recent report of differences in fMRI brain activation to food as a function of genetic subtypes [12]. Furthermore, the correlation be- 
tween GH therapy (at any point during lifetime) and the completion of the fast did not reach statistical significance ( $p=0.07)$. It is of interest, however, that all of the individuals with a history of GH therapy (9/29) completed the fast. Anecdotal reports of changes in appetite or in food-seeking behavior following GH therapy exist but no controlled studies have been published [13,14].

We hypothesized that motivational factors or control mechanisms might correlate with the ability to fast.

1) Motivational factors: Religious motivation and the commitment to other routine religious behaviors did not impact on the ability to fast. This finding might be highly significant and suggests that the capacity to fast may not be limited to a specific religious practice and that the results of this study may be applicable to a wider range of circumstances and populations.

Social motivation including the ability to say "I also fasted" during the post-fast dinner with the family or hostel companions was considered, but again no correlation was found between the social desirability motivational factor and fasting.

Among the motivational factors, only the caretakers' assessment of the subjective importance of the fast for the PWS individual was significant for those who fasted. Although one could argue that the caregivers' expectations affect the ability to fast among PWS individuals, this explanation is unlikely since other wishes and expectations of caregivers are generally disregarded. Therefore our findings could indicate that the main factor that influences the ability to fast to completion is an intrinsic motivation which is intuitively perceived by caregivers but is not identified by any other measure.

2) Control factors: We found that PWS individuals who fasted had a higher threshold to pain and better sense of control. Research shows that when eating, individuals with PWS activate neural substrates similar to those involved in addictions $[15,16]$. The findings of these researchers suggest a new hypothesis, i.e. that the meaning of fasting for PWS individuals might be overcoming the food addiction rather than overcoming hunger. Our results (i.e. that those who successfully completed the fast felt more in control in their eating habits and had a higher threshold to pain than the non-fasters) are in line with this hypothesis: an increased ability to tolerate pain could be similar to an enhanced ability to overcome the reward-seeking tendency.

Control mechanisms may also have been facilitated by the fact that Yom Kippur has a pre-determined time limit and is not an open ended task.

The results of this study could also be seen as support for another recently proposed hypothesis suggesting that overeating in PWS is a consequence of inability to stop eating accompanied by an impaired satiety response [15]. Thus, the ability to fast could be explained by the fact that the individuals in our sample (who were motivated to exert control over initiation of eating) were not challenged with the core dysfunction in PWS, according with the last mentioned hypothesis, of an inability to stop eating.

Holsen et al. state that PWS has the potential to serve as a model for extreme obesity [16]. These authors emphasize that in PWS there are two dysfunctional steps in the mechanism of the drive to eat and eating regulation. One is hyper-activation of the reward, subcortical circuitry in response to food stimuli and the other is less effecttive recruitment of inhibitory, self-control mechanisms, both contributing to excessive overeating. In this study, we have shown that PWS individuals are able, albeit under special circumstances, to overcome these physiological limitations and successfully fast for an extended period. We hypothesize that special circumstances can improve the capacity to activate inhibitory control mechanisms in PWS and possibly in other obese individuals, as well. Behavioral therapeutic protocols employing special motivational circumstances could be created in which obese persons will experience the capacity to fast, control their drive to eat for a much longer period than thought possible, and thereby increase internal awareness of their control mechanisms.

There are some limitations to the study. Although the sample size is relatively small, it is representative of this rare syndrome and includes all the PWS individuals in Israel who were candidates to fast voluntarily on Yom Kippur. The relatively large number of factors studied is also statistically problematic.

Although explicit rewards for fasting were ruled out, it is possible that additional implicit rewards exist, besides those included in the questionnaires.

The subjects were all interviewed prior to the fast which might have functioned as a priming factor. This seems unlikely and at best minor given the national nature and public exposure of the Yom Kippur fast.

Most of the behavioral questionnaires used were not specifically designed and standardized to the PWS population, since no such questionnaires are available.

This prospective study documents that adolescents and adults with PWS can voluntarily abstain from food for 25 hours. Since food restriction is one of the major avenues for treating individuals with PWS, understanding the mechanisms involved in successful fasting could be an important contribution in developing more effective treatment of this syndrome. Our findings suggest that in spite of extreme hyperphagia, PWS individuals are able to activate mechanisms which improve their control of eating for a longer period than expected. The observation that the degree of religiosity did not impact on the ability to fast suggests that these mechanisms may be applicable to a wider range of circumstances and populations. Since 
PWS has the potential to serve as a model for extreme obesity, the ability for self-control under special circumstances deserves further study; it may be relevant to other types of severe obesity and possibly lead to improved methods of behavioral modification.

\section{ACKNOWLEDGEMENTS}

The authors are deeply grateful to Ruth Shalev MD for her thoughtful comments and suggestions in revising the manuscript.

\section{REFERENCES}

[1] Cassidy, S.B. and Driscoll, D.J. (2009) Prader-Willi syndrome. European Journal of Human Genetics, 17, 3-13. doi:10.1038/ejhg.2008.165

[2] Miller, J.L., Lynn, C.H., Driscoll, D.C., Goldstone, A.P., Gold, J.A., Kimonis, V., Dykens, E., Butler, M.G., Shuster, J.J. and Driscoll, D.J. (2011) Nutritional phases in PraderWilli syndrome. American Journal of Medical Genetics A, 155A, 1040-1049. doi:10.1002/ajmg.a.33951

[3] Benarroch, F., Hirsch, H.J., Genstil, L., Landau, Y. and Gross-Tsur, V. (2007) Prader-Willi syndrome: Medical prevention and behavioral challenges. Child and Adolescent Psychiatric Clinics of North America, 16, 695-708. doi:10.1016/j.chc.2007.03.007

[4] Central Bureau of Statistics Social Survey (2009) Jewish tradition observance and changes in religiosity of the Jewish population in Israel.

http://www.cbs.gov.il/www/hodaot2010n/19_10_211e.pd $\underline{\mathrm{f}}$

[5] Karlsson, J., Persson, L.O., Sjöström, L. and Sullivan, M. (2000) Psychometric properties and factor structure of the Three-Factor Eating Questionnaire (TFEQ) in obese men and women. Results from the Swedish Obese Subjects (SOS) study. International Journal of Obesity and Related Metabolic Disorders, 24, 1715-1725. doi:10.1038/sj.ijo.0801442

[6] Derryberry, D. and Rothbart, M.K. (1988) Arousal, affect and attention as components of temperament. Journal of Personality and Social Psychology, 55, 958-966. doi:10.1037/0022-3514.55.6.958

[7] Auerbach, J.G. and Lerner, Y. (1991) Syndromes derived from the Child Behavior Checklist for clinically referred Israeli boys aged 6 - 11: A research note. Journal of Child Psychology and Psychiatry, 32, 1017-1024. doi:10.1111/j.1469-7610.1991.tb01926.x

[8] Gioia, G.A., Isquith, P.K., Guy, S.C. and Kenworthy, L. (2000) Behavior rating inventory of executive function. Child Neuropsychology, 6, 235-238. doi:10.1076/chin.6.3.235.3152

[9] Linder, N., Kroyzer, N., Maeir, A., Wertman-Elad, R. and Pollak, Y. (2010) ADHD and executive dysfunctions, measured by the Hebrew version of Behavioral Rating Inventory of Executive Functions (BRIEF). Child Neuropsychology, 16, 494-502. doi:10.1080/09297041003781884

[10] Gross-Tsur, V., Landau, Y.E., Benarroch, F., WertmanElad, R. and Shalev, R.S. (2001) Cognition, attention and behavior in Prader-Willi syndrome. Journal of Child Neurology, 16, 288-290. doi:10.1177/088307380101600411

[11] Shapiro Jr., D.H., Weatherford, V., Kaufman, E. and Broenen, R.E. (1994) A control profile of adult children of alcoholics: A preliminary investigation. American Journal of Drug and Alcohol Abuse, 20, 247-262. doi:10.3109/00952999409106785

[12] Holsen, L.M., Zarcone, J.R., Chambers, R., Butler, M.G., Bittel, D.C., Brooks, W.M., Thompson, T.I. and Savage, C.R. (2009). Genetic subtype differences in neural circuitry of food motivation in Prader-Willi syndrome. International Journal of Obesity, 33, 273-283. doi:10.1038/ijo.2008.255

[13] Myers, S.E., Carrel, A.L., Whitman, B.Y. and Allen D.B. (2000) Sustained benefit after 2 years of growth hormone on body composition, fat utilization, physical strength and agility, and growth in Prader-Willi syndrome. Journal of Pediatrics, 137, 42-49. doi:10.1067/mpd.2000.105369

[14] Burman, P., Ritzén, E.M. and Lindgren, A.C. (2001) Endocrine dysfunction in Prader-Willi syndrome: A review with special reference to GH. Endocrinology Review, 22, 787-799. doi:10.1210/er.22.6.787

[15] McAllister, C.J., Whittington, J.E. and Holland, A.J. (2011) Development of the eating behaviour in PraderWilli Syndrome: Advances in our understanding. International Journal of Obesity, 35, 188-197. doi:10.1038/ijo.2010.139

[16] Holsen, L.M., Savage, C.R., Martin, L.E., Bruce, A.S., Lepping, R.J., Ko, E., Brooks, W.M., Butler, M.G., Zarcone, J.R. and Goldstein J.M. (2012) Importance of reward and prefrontal circuitry in hunger and satiety: Prader-Willi syndrome vs simple obesity. International Journal of Obesity, 63, 638-647. doi:10.1038/ijo.2011.204 